\title{
Primary care management of carpal tunnel syndrome
}

\author{
F D Burke, J Ellis, H McKenna, M J Bradley
}

Postgrad Med J 2003;79:433-437

Carpal tunnel syndrome of mild to moderate severity can often be effectively treated in a primary care environment. Workplace task modification and wrist splints can reduce or defer referral to hospital for surgical decompression. Nerve and tendon gliding exercises may also be of benefit. Steroid injections to the mouth of the carpal tunnel are particularly useful for symptomatic women in the third trimester of pregnancy. However inadvertent neural injection may cause disabling chronic pain. Referral to a minority of practitioners trained in the technique would ensure sufficient patient numbers to maintain skill levels. high. De Krom et al sought histories compatible with carpal tunnel syndrome in 1000 adults in a community and authenticated the diagnosis in those with the appropriate history by nerve conduction studies. ${ }^{5}$ They found that $3.4 \%$ of adult females were known to have carpal tunnel syndrome with an additional $5.8 \%$ of females in whom the condition had not previously been detected. The prevalence can only be a minimal estimate (some patients with an appropriate history refused nerve conduction studies and were classified as not suffering from carpal tunnel syndrome, despite the appropriate history). Ferry et al performed a similar study in the United Kingdom with a prevalence estimate of between $7 \%$ and $16 \%$, concluding that the condition was common in the general population. ${ }^{6}$

\section{DIAGNOSTIC CRITERIA}

Harrington et al suggested surveillance criteria for carpal tunnel syndrome should be pain or paraesthesia or sensory loss in the median nerve distribution and one of the following ${ }^{7}$ :

- Tinel's test positive.

- Phalen's test positive.

- Nocturnal exacerbation of symptoms.

- Motor loss with wasting of the abductor pollicis brevis.

- Abnormal nerve conduction studies.

Tinel's test (percussion of the median nerve at the wrist creating tingling in the median innervated fingers) is considered to have a specificity of $99 \%$ and a sensitivity of $64 \% .^{8}$ Phalen's test (wrist flexion provoking tingling in median innervated fingers within 60 seconds) has a 95\% specificity with a sensitivity of $75 \%$.

The Levine Questionnaire is considered by many to be a valuable assessment of severity of symptoms and functional status in carpal tunnel syndrome. ${ }^{9}$ The self administered questionnaire is helpful in screening patients for carpal tunnel syndrome and for evaluating the effect of the therapeutic interventions.

\section{ASSOCIATED FEATURES}

Most studies identify a threefold to fourfold preponderance of females to males. The condition is associated with a wide variety of clinical conditions including obesity, rheumatoid arthritis, pregnancy, diabetes, thyroid dysfunction, renal dialysis (amyloid), and radial malunion. The double crush phenomenon may play a part with minor compression at neck and wrist summating to create significant clinical complaints. ${ }^{10} \mathrm{~A}$ minority of patients will present with a strong family history arising from hereditary neuropathy with liability to pressure palsies. ${ }^{11}$ Oral 
contraceptives, or other medications, which tend to cause fluid retention may also provoke carpal tunnel syndrome.

\section{SEVERITY OF SYMPTOMS}

Patients commonly present with numbness and tingling in the median innervated fingers which wakes them at night. Symptoms are mild and infrequent at the onset and may resolve spontaneously for months or years. Futami found that one third of patients with untreated carpal tunnel syndrome had resolved spontaneously when reviewed at six months. ${ }^{12}$ The study included some pregnant females.

Untreated carpal tunnel syndrome commonly gradually deteriorates with increasing frequency of numbness and tingling and sleep disturbance. The tingling becomes unremitting and then progresses to numbness. This change (from constant paraesthesia to numbness) may be perceived by the patient to be an improvement on the basis of reduced discomfort. Weakness and finally wasting of the abductor pollicis brevis is usually a late feature of severe compression.

Prolonged severe compression of the median nerve produces observable flattening of the nerve at the site of decompression with a prestenotic dilatation. This may cause intraneural fibrosis and jeopardise the benefits of nerve decompression, particularly in the most severe cases, giving rise to persistent symptoms.

Mild cases are those with a short history of intermittent episodes of paraesthesia. Moderate cases may be considered those with frequent episodes of paraesthesia or numbness. Severe cases have persistent paraesthesia or numbness or wasting of the abductor pollicis brevis.

\section{PRIMARY CARE TREATMENT OPTIONS Workplace task modification}

The association between carpal tunnel syndrome and work remains controversial. Frequent forceful use of the hands often exacerbates the symptoms of carpal tunnel syndrome. The case for work practices causing carpal tunnel syndrome is less clear, as is the mechanism by which the resting pressure rises in the carpal tunnel. The most frequently used hypothesis is that the use of the hands provokes a flexor tenosynovitis. ${ }^{13}$ However, Kerr et al biopsied the synovium in over 600 cases at carpal tunnel decompression and found acute or chronic synovitis in only $4 \%$ of cases. ${ }^{14}$

Cobb et al demonstrated that lumbrical muscle excursion into the carpal tunnel can raise the pressure and possibly provoke symptoms. ${ }^{15}$ Task modification that reduces metacarpophalangeal joint flexion range will reduce lumbrical incursion and may control symptoms. Heavy manual workers with large lumbricals may be at increased risk of carpal tunnel syndrome if obliged to grip firmly for prolonged periods of time.

Many women develop carpal tunnel syndrome in their fourth or fifth decades and their duties at work may be suggested as the cause. Perusal of their general practitioner records commonly reveals previous episodes of carpal tunnel syndrome in their 20s when pregnant, indicating a constitutional vulnerability, and work in these circumstances would more reasonably be considered an exacerbator of carpal tunnel syndrome, rather than the cause.

Physiotherapists and occupational therapists can offer workers and their employers advice on task modification, which will often control mild or moderate symptoms of carpal tunnel syndrome. The ergonomics of the workplace can be assessed to avoid protracted hand use at extremes of joint range. The position of the wrist during work is crucial in controlling symptoms of carpal tunnel syndrome. The pressure in the carpal tunnel is lowest in neutral wrist position (normal range $0-7 \mathrm{~mm} \mathrm{Hg}^{16}$ ) but swiftly rises if the wrist is moved into flexion or extension. Adjustment of work height or tools can optimise the wrist position and avoid extremes of range. The diameter of tool handles may also be adjusted to minimise grip forces. Tasks may be varied or rotated to spread the most forceful or repetitive activities evenly throughout the shift. Rest breaks should be applied in a similar manner. Often simple and obvious alterations to working practice can be beneficial in controlling milder symptoms of carpal tunnel syndrome.

\section{Nerve and tendon gliding exercises}

Wilgis and Murphy have popularised the concept of some cases of nerve compression arising from an adhesive neuritis, with tethering of the nerve with limited excursion of the nerve through wrist and finger flexion extension range. ${ }^{17}$ The normal excursion of the median nerve at the wrist is approximately $14 \mathrm{~mm}$. Tethering of the nerve reduces the perfusion within the nerve and compromises function. Digital flexor tendon mobilising techniques are particularly valuable in the management of postoperative carpal tunnel patients. ${ }^{18}$ However, the technique has also been shown to be of benefit in patients being considered for surgery. Rozmaryn et al reviewed more than 200 hands under consideration for carpal tunnel decompression. ${ }^{19}$ Altogether $71 \%$ of the patients who were not offered gliding exercises went forward to surgery; only $43 \%$ of the gliding exercise group were felt to require surgery. Care should be taken that the frequency and force of the exercises is not so great that exacerbation of symptoms is provoked. The beneficial effect of gliding exercises may be the mobilisation of the nerve directly or facilitation of venous return or oedema dispersal. Early cases of carpal tunnel syndrome may benefit from the use of this treatment option.

\section{Wrist splints}

The pressure in the carpal tunnel is lowest in neutral wrist flexion extension range, with the pressure rising significantly as the wrist is moved into flexion or extension. ${ }^{20}$ Splints that hold the wrist in neutral are often helpful in controlling symptoms of mild to moderate severity. They can be particularly useful in cases of early carpal tunnel syndrome, when the patient is repeatedly woken at night by painful paraesthesia. $^{21}$ Splints are less effective in cases where there is continuous paraesthesia or numbness. Splints that are a poor fit are unlikely to be used. It is a false economy to simply offer the cheapest available splint. It is helpful to have a range of commercially available prefabricated splints and to provide the most suitable in each case (fig 1). The policy requires the retention of a wider range of splints but ensures improved patient compliance.22 A minority of patients will not find a suitable commercially available prefabricated splint and a custom-made splint can then be applied. Commercially available splints are usually packaged with the wrist bar bent to an extended position. Most recipients of wrist splints are benefited by a moderately extended wrist position (which facilitates grasp). It is not, however, the appropriate position for patients with carpal tunnel syndrome, and their symptoms may well be exacerbated by the raised pressure in the carpal tunnel. The splint should be adjusted to neutral dorsipalmar flexion before fitting.

Crow found splintage to be of considerable benefit in treating mild to moderate cases of carpal tunnel syndrome. ${ }^{23}$ Three quarters of his group benefited and some were able to discontinue splintage without a recurrence of symptoms in the short term.

There is a reluctance to advise continuous splint use and most hand therapists prefer the use of splints at night and only occasionally during the day, if there are particular activities likely to exacerbate symptoms. For optimal effect splints should be chosen and fitted by experienced hand therapists from a range of available options, with the opportunity for a further visit to audit outcome and allow for additional adjustments. The interviews also permit a discussion on task modifications at work and home as previously described. The therapist will also have access to surgeons if such a referral is felt to be necessary. 


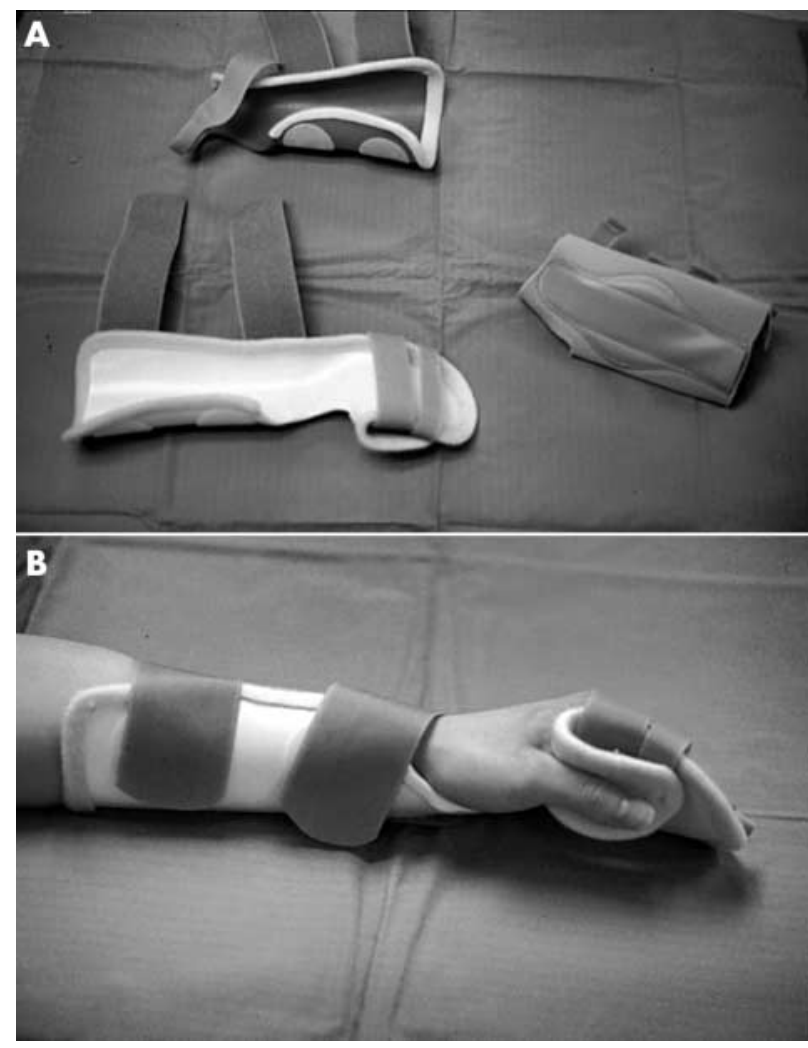

Figure 1 Splints offered to patients in a hand therapy primary care clinic.

The Daytimer Splint is a useful addition to the brace range, particularly if there is poor compliance or the daytime symptoms are intrusive. The splint consists of a wrist strap and adjustable loop on the dorsal surface, which extends around the base of the middle finger. The loop is shortened until the wrist is unable to flex beyond the neutral point (fig 2). Wrist posture is therefore adjusted with minimal obstruction to the surface of palm or fingers. The splint can be useful in re-educating a patient's wrist and hand posture with minimal interference to use.

\section{Steroid injections}

Steroid injection directly into the carpal tunnel is unnecessary and undesirable. Steroid (with some local anaesthetic) may reasonably be inserted either to the radial or ulnar side of the median nerve, just proximal to the wrist crease. A position midway between palmaris longus and the flexor carpi ulnaris is probably the most frequently used site. Disproportionate pain or symptoms referred to the median innervated fingers require immediate adjustment of the position of the needle. Inadvertent injection of depot steroid into the median nerve is potentially disastrous to hand function. It may leave a chronic disabling paraesthesia. ${ }^{24}$ It therefore seems prudent for a minority of general practitioners to gain experience in the technique and provide the service for a group of colleagues, thereby avoiding a situation where all practitioners only very occasionally give an injection to this somewhat hazardous area.

Steroid injections are very effective in resolving symptoms of carpal tunnel syndrome, in the short term, particularly if symptoms are mild and intermittent. ${ }^{25}$ The short term efficacy is so high that the injection can be used as a test to discriminate in the diagnostic dilemma of a possible carpal tunnel syndrome or referred pain from the cervical spine. Temporary benefit after a steroid injection at the mouth of the carpal tunnel is strongly suggestive of a diagnosis of carpal tunnel
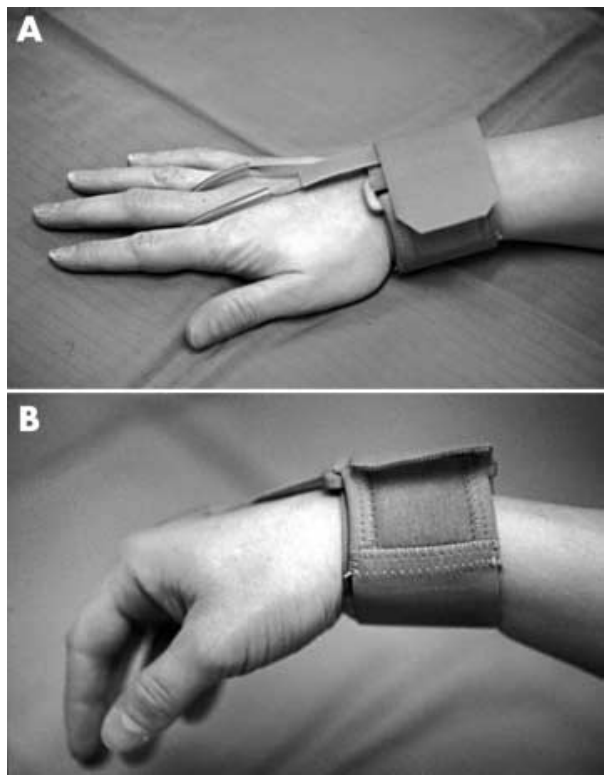

Figure 2 (A) The Daytimer Splint showing loop around middle finger. (B) The loop permits only limited wrist flexion yet offers minimal obstruction to the palm and fingers.

syndrome in such circumstances. Gelberman et al observed that $22 \%$ of patients were still symptom-free 18 months after a steroid injection, with symptoms recurring to a greater or lesser degree in the remainder. ${ }^{26}$ Those with mild symptoms of short duration had a more abiding benefit than those with more protracted or severe symptoms.

The most obvious indication for a steroid injection in the management of acute carpal tunnel syndrome is those presenting in the third trimester of pregnancy. Injection will commonly resolve the situation for a sufficient length of time to take the patient through to delivery (when symptoms frequently abate).

\section{Ultrasound}

There is limited evidence on the effectiveness of ultrasound in relieving the symptoms of carpal tunnel syndrome. Ebenbichler et al reviewed a small group of patients and found the treatment to be beneficial in mild to moderate cases. ${ }^{27}$ The benefit was observed symptomatically and confirmed neurophysiologically with nerve conduction studies. Oztas et al offer a more cautious view. ${ }^{28}$ Their study did not show a benefit and questioned the effect of ultrasound on an already damaged nerve (their post-treatment nerve conduction studies showed some reduction in function).

\section{ALTERNATIVE MODALITIES OF TREATMENT}

Diuretics were commonly used in the past to either treat or diagnose carpal tunnel syndrome. ${ }^{29}$ Their use has been superseded by a depot steroid injection around the mouth of the carpal tunnel, which appears at the least equally effective with less systemic side effects. Vitamin B12 (pyridoxine), was offered as a therapeutic agent with nerve healing properties (partly on the basis of B12 deficiency causing neuropathy). Amadio considered its benefit to be minimal with low evidence of effectiveness. ${ }^{30}$ Non-steroidal anti-inflammatory medications may be of some benefit in carpal tunnel syndrome, associated with an acute inflammatory process, but their effect in most cases of carpal tunnel syndrome presenting for treatment is slight.

Garfinkel et al recommend yoga as a helpful intervention, with improvements in grip strength and pain reduction. ${ }^{31}$ Yoga was trialled against the use of a wrist splint. Eleven yoga postures were chosen to strengthen, stretch, and balance joints 
in the upper body with relaxation for a period of eight weeks Several of the exercises involved mobilisation of shoulders and neck, with other involving wrist and hand. Sleep disturbance was not improved nor were there improvements in nerve conduction studies. The use of yoga is in keeping with the view that upper limb neurological complaints can often be eased by improved posture and a general aerobic exercise programme. ${ }^{32}$

\section{REFERRAL TO HOSPITAL}

Many early cases of carpal tunnel syndrome with intermittent symptoms will be controlled in the short to middle term by conservative means, previously described. Failure to respond to conservative measures provokes consideration of a referral to hospital. Studies of failure of attendance at hand clinics reveals a relatively high incidence in patients with carpal tunnel syndrome $(28 \%)$ and ganglia $(20 \%)$.

Bradley studied reasons for non-attendance in new patient hand clinics and found (contrary to expectation) that patients who had had a good explanation of the condition from their general practitioners were more likely to fail to attend than those who had received a poor explanation. ${ }^{33}$ Knowledge empowers patient choice; it allows them to balance the competing pressures in their lives, including the option of not attending the clinic if reassured by the advice offered by the general practitioner. Failure to attend in an "informed patient" may indicate that they may not have been a full partner in the decision to refer. Alternatively they may have subsequently changed their mind due to employment or financial pressures which often present barriers to attendance. In-depth interviews with patients indicated the need for the general practitioner to ensure the patient was aware that referral implied the likelihood of an operative procedure being performed to the hand. It is helpful if the general practitioner initiates the consent process with basic details of carpal tunnel decompression, including the postoperative recovery period. Haywood et al designed a protocol for referral from primary care based on severity of symptoms and prognostic indicators of outcome with conservative treatment. ${ }^{34}$ The protocol stipulates that (if referred) the patient must be aware that surgery may be necessary.

\section{CARPAL TUNNEL DECOMPRESSION}

Carpal tunnel decompression is an effective means of relieving symptoms not controlled by conservative means. The operation is usually performed as a day case under local infiltration anaesthesia. The limb is prepared and draped and exsanguinated with a sterile crepe bandage. The pressure cuff to arm is then inflated above systolic pressure to create a bloodless field at the wrist. The transverse carpal ligament is fully released, the skin stitched, and a crepe and cotton wool dressing applied. The tourniquet is released and the patient returned to the ward with the limb raised. The tourniquet time does not usually exceed 15 minutes. The patient is usually suitable for discharge within an hour of return to the ward. Sutures are removed 10-14 days from surgery and firm grip should be avoided for six weeks. Physiotherapy is rarely required. Release of the transverse carpal ligament increases the volume of the carpal tunnel by $15 \%-20 \%$.

A local audit of over 200 carpal tunnel decompressions revealed two thirds were cured, with a quarter of cases left with minor symptoms. ${ }^{35}$ Less than $10 \%$ of cases are left with significant ongoing symptoms (usually persistence of symptoms or a tender scar). It is unusual for patients to be worse after surgery. Failure to benefit from decompression may arise from concomitant disease (for example diabetes) or as a double crush phenomenon with additional nerve root irritation at cervical spine level. Patients who have experienced severe prolonged compression may have intraneural fibrosis with a more limited recovery postoperatively.
Box 1: Key points concerning the primary care management of carpal tunnel syndrome

- Carpal tunnel syndrome is often a progressive condition but the symptoms are commonly fluctuent in nature in the short to middle term and amenable to conservative management in primary care.

- Patients with mild to moderate symptoms of carpal tunnel syndrome often have a satisfactory response to work modification or conservative management.

- Steroid injection provides effective temporary relief but symptoms are likely to return within a few months. The treatment, however, is particularly effective for pregnant patients, near delivery, as carpal tunnel syndrome is likely to resolve after pregnancy.

- Inadvertent steroid injection into the median nerve is a significant hazard, which may well give rise to chronic pain states. Those considering steroid injections around the mouth of the carpal tunnel should be familiar with the course of the median nerve.

- Splintage with the wrist in a neutral position, at night or during the day, will commonly control symptoms in patients with intermittent numbness and tingling in the short to middle term, at least.

- Patients with persistent symptoms are less likely to be relieved by conservative means.

- If a patient is being referred for surgery they should be aware of the general character of the process and agreeable to having surgery, if it is deemed necessary.

\section{Authors' affiliations}

F D Burke, J Ellis, H McKenna, M J Bradley, Pulvertaft Hand Centre, Derbyshire Royal Infirmary, Derby

\section{REFERENCES} 1 Burke FD, Dias JJ, Bradley M, et al. Providing care for hand
disorders - the Derby Hand Unit experience 1989-90. J Hand Surg [Br] $1991 ; 16: 13-18$.

2 Burke FD. Carpal tunnel syndrome-reconciling “demand management" with clinical need. J Hand Surg [Br] 2000;25:121-7.

3 Stevens JC, Sun S, Beard CM, et al. Carpal tunnel syndrome in Rochester, Minnesota 1961-1980. Neurology 1988:38:134-8.

4 Keller RB, Largay AM, Soule DN, et al. Maine carpal tunnel study: small area variations. J Hand Surg [Am] 1998;23:692-6.

5 De Krom MC, Knipschild PG, Kester AD, et al. Carpal tunnel syndrome prevalence in the general population. J Clin Epidemiol 1992;45:373-6.

6 Ferry S, Prichard T, Keenan J, et al. Estimating the prevalence of delayed median nerve conduction in the general population. $\mathrm{Br} J$ Rheumatol 1998:37:630-5.

7 Harrington JM, Carter JT, Birrel L, et al. Surveillance case definitions for work related upper limb pain syndromes. Occup Environ Med 1988;55:264-71

8 Szabo RM, Slater RR, Farvery TB, et al. The value of diagnostic testing in carpal tunnel syndrome. J Hand Surg [Am] 1999;24:704-14.

9 Levine DW Simmons BP, Koris M, et al. A self administered questionnaire for the assessment of severity of symptoms and functional status in carpal tunnel syndrome. J Bone Joint Surg Am 1993;75: 1585-92

10 Hurst LC, Weissberg D, Carrol RE. Relationship of the double crush to carpal tunnel syndrome. An analysis of 1000 cases of carpal tunnel syndrome. J Hand Surg [Br] 1995:10:202-4.

11 Lane JE, Foulkes GD, Hope TD, et al. Hereditary neuropathy with liability to pressure palsies mimicking multifocal compression neuropathy. $J$ Hand Surg [Am] 2001;26:670-4.

12 Futami T, Kobayashi A, Ukita T, et al. Carpal tunnel syndrome-its natural history. Hand Surgery 1997;2:129-30.

13 Von Schroeder HP, Botte M. Carpal tunnel syndrome. Hand Clinics 1996: 12:643-55

14 Kerr CD, Sybert DR, Albarracin NS. An analysis of the flexor synovitis in idiopathic carpal tunnel syndrome. J Hand Surg [Am] 1992;17:102830.

15 Cobb TK, Kai Nan A, Cooney WP. Effect of lumbrical muscle incursion within the carpal tunnel on carpal tunnel pressure: a cadaveric study. $J$ Hand Surg [Am] 1995;20:186-92.

16 Gelberman G, Hergenroeder PT, Hargens AR, et al. The carpal tunnel syndrome. A study of carpal canal pressures. J'Bone Joint Surg Am $1981 ; 63: 380-3$. 
17 Wilgis EFS, Murphy R. The significance of longitudinal excursion in peripheral nerves. Hand Clinics 1986:2:761-6.

18 Totten PA, Hunter JM. Therapeutic techniques to enhance nerve gliding in thoracic outlet syndrome and carpal tunnel syndrome. Hand Clinics $1991 ; 7: 505-20$.

19 Rozmaryn LM, Dovelle S, Rothman ER, et al. Nerve and tendons gliding exercises and the conservative management of carpal tunnel syndrome. $J$ Hand Ther 1998:11:171-9.

20 Gelberman RG, Hergenroeder PT, Hargens AR, et al. The carpal tunnel syndrome. A study of carpal canal pressures. J Bone Joint Surg Am 1981;63:380-3.

21 Sailer SM. The role of splinting in rehabilitation in the treatment of carpa and cubital tunnel syndromes. Hand Clinics 1996;12:223-41.

22 Stern EB, Sines B, Teague TR. Commercial wrist extensor orthoses. Hand function comfort and interference across five styles. J Hand Ther 1994; 7:237-44.

23 Crow RS. Treatment of carpal tunnel syndrome. BM 1960;i:1611-15.

24 Tavares SP Giddins GEB. Nerve injury following steroid injection for carpal tunnel syndrome. J Hand Surg $[B r]$ 1996;21:208-9.

25 Babu SR, Britton JM. The role of steroid injections in the management of carpal tunnel syndrome. Journal of Orthopaedic Rheumatology 1994;7:59-60.
26 Gelberman RH, Aronson D, Weisman MH. Carpal tunnel syndrome: results of a prospective trial of steroid injection and splinting. J Bone Joint Surg Am 1980:62:1181-4.

27 Ebenbichler GR, Resch KL, Nicolakis P, et al. Ultrasound treatment for treating the carpal tunnel syndrome. Randomised "sham" controlled trial. BM 1998;316:731-5.

28 Oztas O, Turan B, Bora l, et al. Ultrasound therapy effect in carpal tunnel syndrome. Arch Phys Med Rehabil 1998;79:1540-4.

29 Guly PJ. Carpal tunnel syndrome. BM 1959;i: 1184.

30 Amadio PC. Pyridoxine as an adjunct treatment in carpal tunnel syndrome. J Hand Surg [Am] 1978;10:237-41.

31 Garfinkel MS, Singhal A, Katz WA, et al. Yoga based intervention for carpal tunnel syndrome. JAMA 1998;280:1601-3.

32 Sailer SM. The role of splinting in rehabilitation in the treatment of carpal and cubital tunnel syndromes. Hand Clinics 1996;12:223-41.

33 Bradley MJ. Study to investigate the causes of non attendance at hand surgery out-patient clinic for a new-patient appointment. Derby: University of Derby, 1999. (MSc Thesis.)

34 Haywood AC, Bradley M, Burke FD. Primary care referral protocol for carpal tunnel syndrome. Postgrad Med J 2002;78:149-52.

35 Burke FD, Dias JJ, Webster H. Median nerve compression syndrome at the wrist. In: Hunter J, Schneider LH, Mackin EJ, eds. Tendon and nerve surgry in the hand. "A third decade". St Louis: Mosby, 1997.

\section{IMAGES IN MEDICINE.}

\section{Chronic bullous dermatosis of childhood}

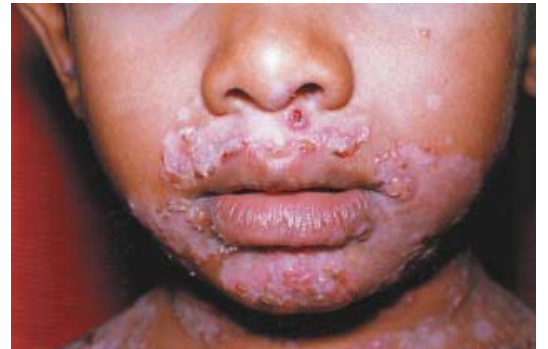

Figure 1 Perioral lesions of chronic bullous dermatosis of childhood (photos published with permission).

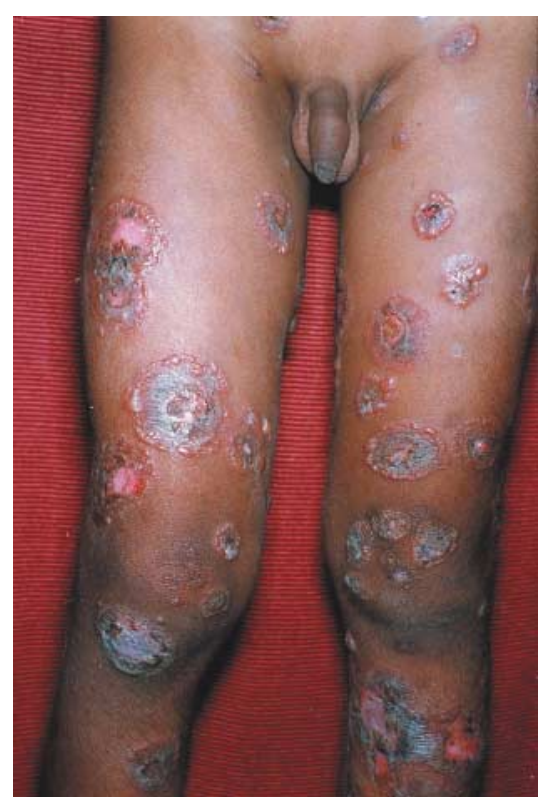

Figure 2 Typical arrangement of vesicles and bullae in an annular or rosette pattern around a central crust- "string of pearls" appearance.
6 year old boy, born of second degree consanguineous parents, presented with mildly itchy blisters all over his body of 20 days' duration. The skin lesions were present for 3-4 days and then ruptured spontaneously. Most of these lesions were healing spontaneously, but a ring of blisters was appearing again around the healed lesions. The child had no preceding history of drug intake and had achieved normal milestones and was vaccinated up to date. There was no family history of a similar disorder. The boy was healthy looking on physical examination, with numerous tense vesicles and bullae on a slightly erythematous skin with erosions and crusting found bilaterally, but asymmetrically on the face, especially in the perioral area (fig l), neck, forearms, thighs (fig 2), groin, and gluteal region (around the anus and perigenital skin). In most of the locations, these vesicles and bullae were arranged in an annular or rosette pattern around a central crust or an erythematous plaque giving the appearance of a "string of pearls" appearance (fig 2).

Routine investigations of haematological and biochemical parameters were within normal limits. Tzanck smear examination from the skin lesions showed neutrophils only; no acantholytic cells were seen. A Gram stained smear of vesicle fluid demonstrated pus cells, but no bacterial organisms were seen. Histopathological examination of a representative biopsy specimen (back) revealed a subepidermal bulla with plenty of neutrophils. The papillary dermis had an accumulation of neutrophils. These findings were consistent with chronic bullous dermatosis of childhood (CBDC). Direct immunofluorescence study of the perilesional skin (back) demonstrated linear deposits of IgA and C3 at the dermoepidermal junction, confirming our clinical diagnosis of CBDC. The child was started on dapsone at a dose of $50 \mathrm{mg}$ per day. Since new lesions were still appearing even after one week of treatment, the dose of dapsone was increased to $50 \mathrm{mg}$ twice a day. He started responding to treatment symptomatically and the lesions completely resolved by 10 days. The child is being followed up.

CBDC is a rare, chronic acquired subepidermal blistering disease of childhood. Among acquired immunobullous diseases in children, the most commonly seen is CBDC and has homogeneous linear deposits of IgA at the epidermal basement membrane. Histopathological examination reveals a subepidermal bulla with collections of neutrophils along the basement membrane, often accumulating at the papillary tips. It predominantly occurs in preschool children (average age of onset is 5 years). The clinical presentation of CBDC is most often characterised by abrupt onset of pruritic or non-pruritic tense vesicles or bullae that can arise on normal or erythematous skin. Most often these lesions occur in clusters giving a "cluster of jewels" or "string of pearls" appearance. These lesions have a predilection for perioral, lower trunk, buttocks, thighs, and perineal surfaces. Oral lesions may occur. The disorder usually remits by age 6-8 years, in most cases by adolescence. The differential diagnosis of CBDC includes bullous impetigo, dermatitis herpetiformis, bullous pemphigoid, and scabies. Paediatricians frequently misdiagnose CBDC as impetigo. In patients with impetigo, Gram stain should demonstrate leucocytes and bacterial organisms.

CBDC is an IgA mediated disorder characterised by IgA antibodies against two different epidermal and dermal associated antigens with molecular weights of $285 \mathrm{kDa}$ and 97/120 kDa. The majority of cases of CBDC respond to either sulfapyridine or dapsone therapy. Dapsone is begun at $\mathrm{l}-2 \mathrm{mg} / \mathrm{kg}$ per day increasing at weekly intervals until symptoms are adequately controlled. The dosage of dapsone should not exceed 3-4 mg/kg per day. Recommendations for the follow up of patients on dapsone are: (1) complete blood cell count and liver function tests at baseline, (2) a complete blood cell count weekly for the first month, monthly for next five months, and every six months thereafter, and (3) liver function tests every six months. Addition of systemic steroids may be required in some cases where there are mixed immunobullous characteristics.

D M Thappa, B Jeevankumar Department of Dermatology and Sexually Transmitted Disease, JIPMER, Pondicherry, 605006, India; dmthappa@jipmer.ed 